\title{
A Lane Centerline Recognition System Based on Improved High Efficiency Hough transform
}

\author{
Tao Peng, YoungMin Jang \\ Department of Electrical Engineering, \\ University of Ulsan, Korea,
}

ORCID: 0000-0002-1155-9630

\author{
ByeongWoo Kim \\ Department of Electrical Engineering, \\ University of Ulsan, Korea,
}

\begin{abstract}
Lane detection is a vital part of the intelligent vehicle detection system. To accurately identify the lane line and improve the real-time and robustness of the lane line detection algorithm, many improvements have been proposed. In this paper, to improve the lane recognition rate more than the conventional method, we preprocess the collected images first, include image grayscale, Sobel operator edge detection and binarization process to obtain high-quality images. Secondly, the improved Hough transform is used to identify and optimize the centerline, to improve the accuracy of the detection results and promote the orderly of detection. The experimental results show that the improved algorithm can identify centerlines more accurately, and has strong real-time, robustness and accuracy.
\end{abstract}

Keywords: Centerline; ROI; Edge Detection; Binarization; Hough transform.

\section{INTRODUCTION}

Autonomous driving technology is the future development trend in the automobile industry. As an important reference mark, the safe driving of smart cars, the lane line is particularly important for accurate detection.

According to relevant research findings, in road traffic accidents, about half of the traffic accidents are related to laneline deviation or related factors. Research shows that if the driver's reaction time can be advanced by 0.5 seconds, at least $60 \%$ of rear-end collisions can be avoided Car crashes, $50 \%$ of cross-collisions and $30 \%$ of head-on collisions. Therefore, improving the technical level of lane centerline detection, perfecting the intelligent vehicle safety assistance system, and reducing the safety hazards in the driving process of the vehicle are of great significance to the family, the enterprise, and even the social development.

Lane centerline detection is the prerequisite for vehicle yaw warning. During vehicle driving, the road environment is complex and changeable. Vehicle driving is not only affected by weather and roadside trees, but also by surrounding vehicles.
These factors will seriously affect the speed and effect of lane centerline detection. At the same time, the detection of the centerline of the lane still has the problem of large amount of calculation and a long time.

In order to solve the above two problems, researchers have conducted extensive research on this. At present, the visionbased lane-line departure system mainly has the following two methods: feature-based recognition method and model-based recognition method. The feature-based recognition method is to use the gray features of the road boundary and lane markings to complete the recognition of the lane markings from the serial gray images in front of the vehicle; the modelbased recognition method is mainly based on different road image models Different recognition technologies are used to recognize lane markings. This paper mainly adopts the featurebased recognition method, which can effectively reduce the complexity of the algorithm and improve the execution speed of the algorithm. Reference[1] proposed to use different methods to identify different scenarios. Although it can be identified in a variety of complex scenarios, a variety of algorithms need to be involved in the identification, so it is impossible to achieve real-time recognition in practical applications. Detection requirements; reference[2] proposed a night lane line detection method, but the algorithm takes a long time; reference[3] used Hough transform to recognize lane lines, but Hough transform is computationally intensive and vulnerable to environment The shortcomings of interference and low detection accuracy have not been completely resolved. Based on existing research, this paper used improved Hough transform to identify and detect centerlines. And optimize the methods in each link to improve the real-time, robustness and accuracy of lane-line detection algorithms.

\section{DESIGN OF THE CENTERLINE RECOGNITION SYSTEM}

\section{The lane model on the road.}

The centerline is an important feature line in the geometric design of road routes, as well as an important control line in road traffic management. In the case of traffic management, 
this is generally used to distinguish the marking line of the two-way vehicle driving space. These lines are mainly marked with yellow lines. The various forms of central lines such as the single solid line, double solid line, single dashed line, double dashed and solid lines as shown in Fig 1 below.

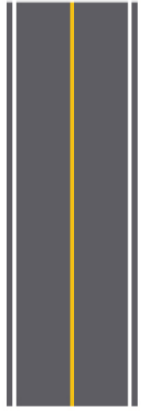

(a) (b)

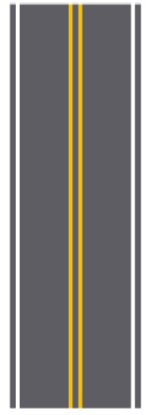

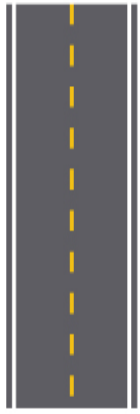

(c)

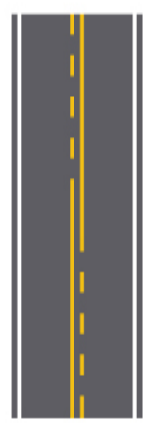

(d) (a). Solid lane: Lanes restricting lane changes and parking.

(b). Solid lanes: The lane he has laid out in two lines to emphasize the meaning of the restriction.

(c). Dashed Line: Lanes Allowed changing, entering, passing.

(d). Solid and Dashed Line: Vehicles on the dashed line may be changed by vehicle; lanes on the solid line are prohibited.

Fig.1 Different type of centerline and the rule.

In road traffic management, different forms of lines have different functions, and the same is true for the centerline. The solid line is strictly forbidden to cross, and the dashed line can be utilized to overtake or turn around while ensuring safety[4].

As show in Fig 1, the lane centerline is divided into the single solid line(Fig 1(a), double solid lines(Fig 1(b), the single dashed line(Fig 1(c) and double dashed and solid line(Fig 1(d), and centerline has a role as shown in the following Fig 1. The system not only need to detect the solid line part, also detect the dashed line part. This is generally difficult and for increase the accuracy of detection in such cases, we have added color recognition to the system.

During the driving process of the vehicle, the front camera is used to capture the lane information image, and the model is built as shown in the fig 2 , In the lane model, $X-Y-Z$ is a coordinate of the road, $x^{\prime}-y^{\prime}-z^{\prime}$ is the coordinates of the input image. The $\mathrm{x}$ accepts as length, $\mathrm{y}$ accepts as width.

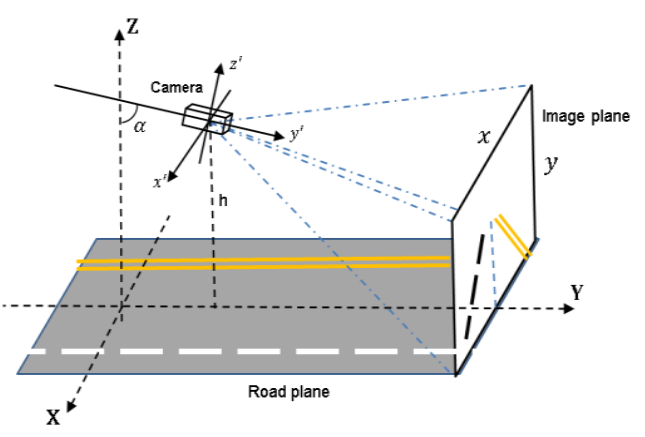

Fig.2 The lane model in input image on the road.

\section{The Centerline recognition algorithm.}

The centerline recognition algorithm is divided into two parts: image preprocessing and centerline detection. Image preprocessing is to process the acquired images through various methods, remove the noise generated in the image acquisition, transmission and processing, filter out the available information in the image, and improve the efficiency of centerline detection. Finally, the improved Hough transform is used to detect the preprocessed image to get the centerline. The above processing will improve the real-time, robustness and accuracy of the centerline detection algorithm, and further improve the vehicle departure warning system. The flow of the centerline recognition system is shown in Fig 3.

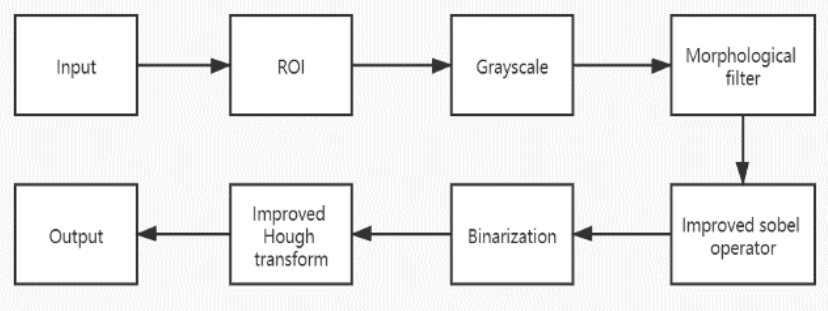

Fig.3 Flowchart of the centerline recognition algorithm.

In order to improve the real-time, robustness and accuracy of the centerline recognition system, the main improvement of this paper is use the morphological filter, an improved sobel operator to identify the edges of features, and an improved Hough transform to fit straight lines. According to the above process, obtains the original image through the camera, and after optimization processing, the real-time processing speed of 27 frames per second is reached. At the same time, the accuracy of processing in different scenarios is $97.82 \%$, which can be adapted to various scenarios.

\section{Region of interest selection.}

In the input image, in addition to the road used to extract the central line, there are unnecessary parts, such as the foreground and the surrounding environment of the road. The general algorithm is applied to the entire image, resulting in calculations processing areas that do not need to be calculated. Makes the processing speed slower. For compensate for this problem, only the partial area required for the center line recognition is selected as the region of interest (ROI). In this article, only the upper and lower regions on the left side of the vehicle forward image are set as ROIs.

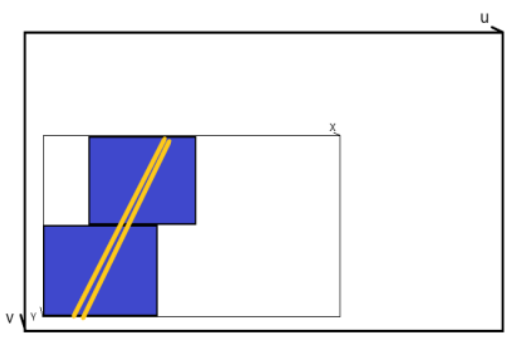

Fig.4 Set operational areas through two ROI. 
Set two sub-regions in the image represented in $(\mathrm{x}, \mathrm{y})$ coordinates where the central line needs to be detected, as shown in Figure 4. Each sub-algorithm has a clockwise rotation based on the upper left corner. Divided into LH-ROI (left high ROI) and LL-ROI (left low ROI). By applying the algorithm proposed in this article under the setting area, parallel processing in each area.

\section{Grayscale and morphological filter.}

After the region of interest is selected, Perform grayscale processing. Color images have three components, R, G, and $\mathrm{B}[5]$, while grayscale images have only one component. Compared with color images, gray-scale images occupied less space and less calculation, which can effectively reduce the burden on the processor and speed up processing efficiency. The gray-scale processing of an image is to convert a color image into a gray-scale image. The conversion methods include component method, average method and weighted average method. For satisfy the rationality of the algorithm, we use the weighted average method to grayscale the image. In the specific weighted calculation, the coefficients corresponding to the three components of $\mathrm{R}, \mathrm{G}$, and $\mathrm{B}$ is $0.3,0.59$, and 0.11 respectively. The calculation equation is as follows:

$$
Y=0.3 R+0.59 G+0.11 B
$$

By equation (1), the grey of the image can be calculated and get the grayscale image.

After grayscale, the image will be filtering. Erosion and dilation[4] is a kind of image morphological filtering, which is to achieves the erosion and dilation of the target by defining a fixed-size structural element for AND operation and OR operation. Dilating is the process of merging all the background points in contact with the object into the object and expanding the boundary to the outside, which can be used to fill the void in the object. Eroding is a process of eliminating boundary points and shrinking the boundary to the inside. Can be used to eliminate small and meaningless objects. This paper used the process of eroding first and then dilating. It is used to eliminate small objects, separate objects at slender points, and smooth the boundaries of larger objects without changing their area significantly. The result of image grayscale and morphological filter is shown in Fig 5.

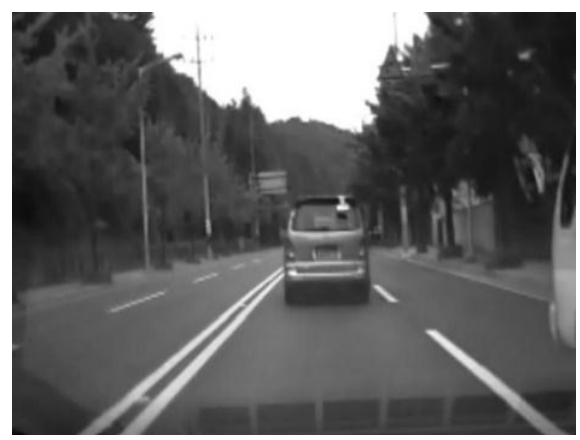

Fig.5 The result after grayscale and filter.

\section{Improved Sobel operator.}

When a vehicle is driving normally on the road, the acquired driving image generally has a strong edge. To obtain the lane centerline, further edge detection is required after image preprocessing, and the related edge detection can be performed by edge extraction. There are many existing edge extraction methods, include Sobel[7], Laplace, Roberts, Canny, etc. Because the Sobel operator is relatively easy to implement in space. And can handle images with gradual grayscale and more noises, and combined with real-time requirements, this paper selects Sobel operator for edge detection. Traditional Sobel operator mostly use two operators in horizontal and vertical directions. In view of the fact that most of the lane lines observed at ordinary times have a certain slope in the image, this paper used the $45^{\circ}$ Sobel operator to extract the edge of the image, The improved template is shown below.

$$
\left[\begin{array}{ccc}
2 & 1 & 0 \\
1 & 0 & -1 \\
0 & -1 & -2
\end{array}\right],\left[\begin{array}{ccc}
0 & 1 & 2 \\
-1 & 0 & 1 \\
-2 & -1 & 0
\end{array}\right]
$$

\section{Image binarization processing.}

After edge detection, there is still a lot of irrelevant information in the image. To further eliminate the irrelevant information, the image needs to be binarized. The key part of the binarization process is the determination of the optimal threshold. Unreasonable determination of the optimal threshold will seriously affect the quality of the image, because when the optimal threshold is set too high, some lane line edge points will be missed, affecting Detection quality: When the optimal threshold is too low, some useless information will be detected, which will affect the detection efficiency.

There are many methods for selecting the best threshold, including the average value of the bimodal method, the maximum between-cluster variance method, the onedimensional maximum Bing method, the minimum value method, and the iterative method. Because the maximum between-class variance method is convenient to calculate, fast in operation, and meets the real-time requirements, this paper selects the maximum between-class variance method to determine the optimal threshold. The maximum between-class variance method is to find the maximum between-class variance between background and foreground. The larger the between-class variance, the greater the difference between the two parts. The effect after sobel and binarization is shown in Fig 6.

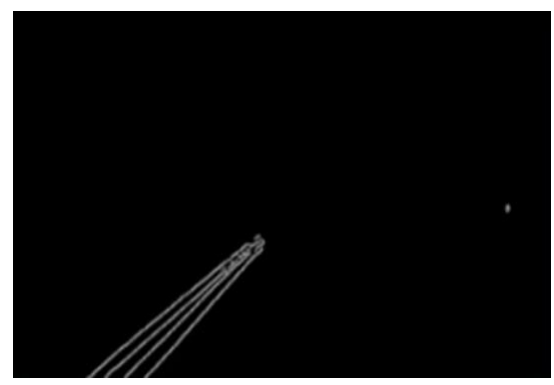

Fig.6 The result after sobel and binarization. 


\section{Hough Transform.}

In the existing research, the acquisition of road information is mainly achieved through monocular vision, but the road environment in real life is more complicated[8], and the acquired images may exist due to road obstacles or street lighting problems and other related reasons. The problem is that it is difficult to obtain continuous edge characteristics. In order to alleviate the image edge discontinuity caused by the above problems, this paper adopts the Hough transform[9-10] method for lane line inspection, because the main advantage of the Hough transform is that it is less affected by noise and curve discontinuities.

\subsection{Ordinary Hough transform algorithm}

Hough transform is an algorithm for detect straight lines, curves and other simple shapes in the image[11]. In this paper, the outer edge of the lane printed through the hough transform is extracted to one line and the centerline is detected. It expresses all the straight lines in the area in manner as shown in equation (2).

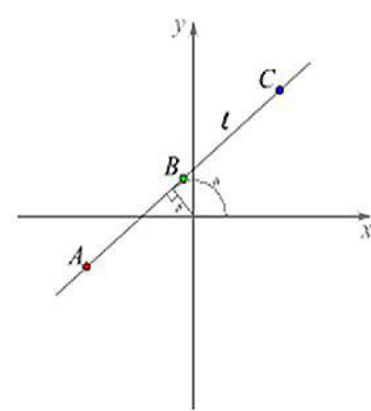

(a) in $x-y$ space

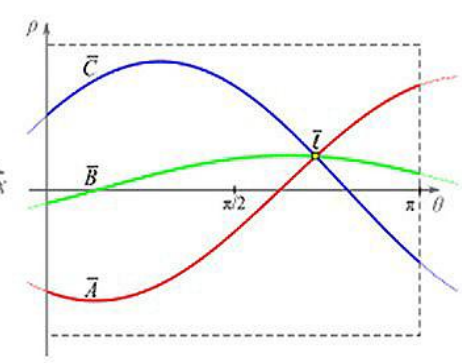

(b) in hough space
Fig.7 Hough transform algorithm.

$$
\rho=x \cos \theta+y \sin \theta
$$

The value $\rho$ is the distance of the line along the vector perpendicular to the line from the origin, and $\theta$ is the $\mathrm{x}$-axis and the angle at which this vector is formed. Hough transformations are expressed as curves in the hough area by generating parameters of $\theta$ and $\rho$ through the expression above, which is in $(\mathrm{x}, \mathrm{y})$ planes.

The straight lines expressed by equation (2) are shown in planes (x,y) as shown in Fig 7(a). $x$ is the horizontal axe and $y$ is the vertical axe in the respectively of image. Points $\mathrm{A}, \mathrm{B}$, and $\mathrm{C}$ are located in the straight line above expressed in Fig 7 (b) as curves in the form of a sinusoidal wave, and straight $\bar{l}$ is expressed as points crossed by curves in the hough area, which are called cumulative cell.

The traditional method of calculating all the values of $\theta$ to find all the straight lines in the image is difficult to recognize, because it extracts all the straight lines shown at the front of the vehicle as well as the boundary of the lane. To solve this problem, an algorithm optimized cumulative cell in the hough area was used[12], and in this paper, the algorithm is applied to the ROI to increase recognition efficiency.

\subsection{Improved Hough transform algorithm.}

Different with conventional algorithms, detection is only performed separately for each ROI. Fig 8 shows the sequence of the proposed Hough transform algorithm. First, when one frame of the image is read into the ROI, algorithm operation is performed at the same time between LLB (lower left border) and HLB (high left border).

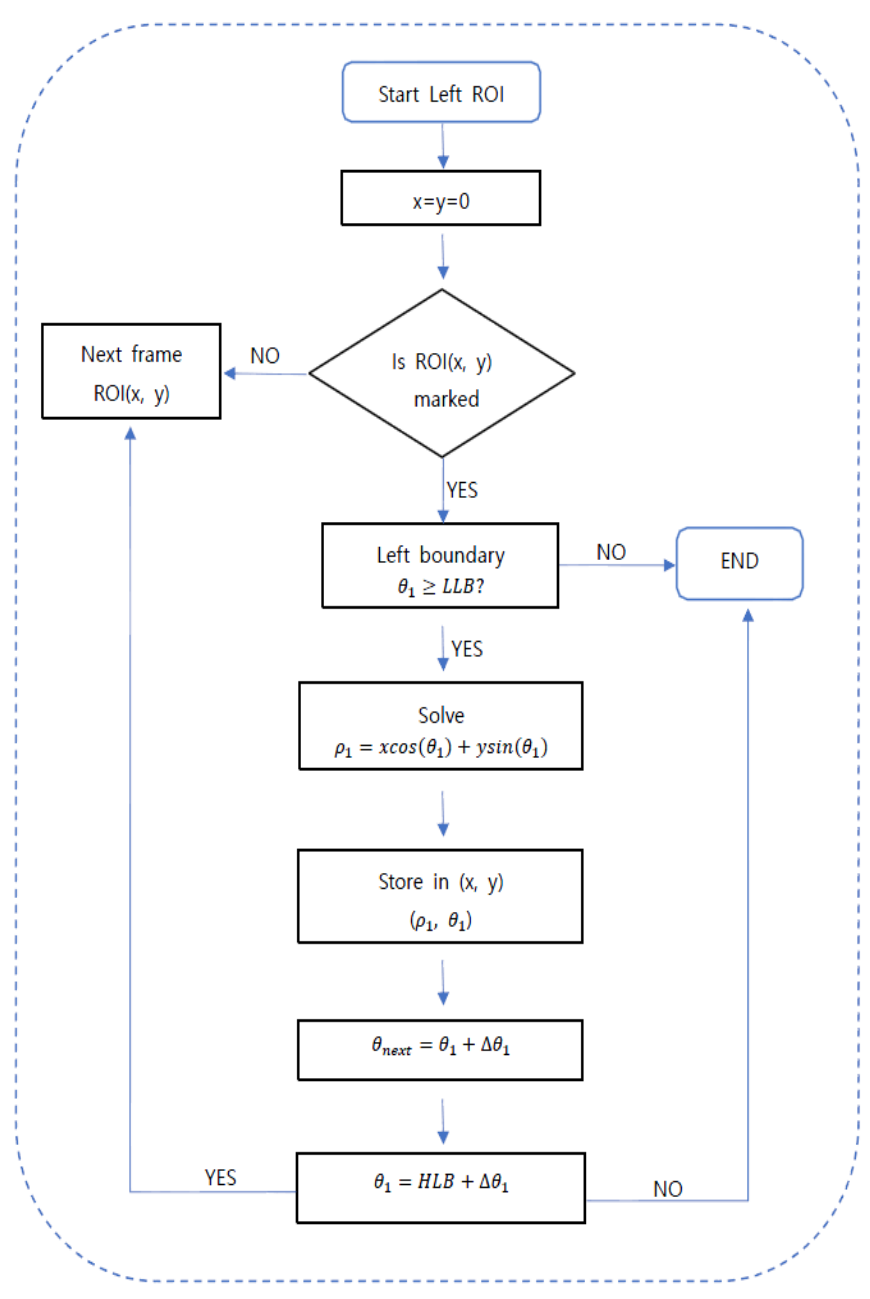

Fig.8 Flowchart of the proposed hough transform algorithm.

For ROI (LL-ROI, LH-ROI), if $\theta_{1}$ is greater than LLB in that area, the value $\rho_{1}$ is calculated using equation (2). Store these $\theta_{1}$ and $\rho_{1}$ in the $(\mathrm{x}, \mathrm{y})$ area and output corresponding detected lines. $\theta$ next, the $\theta$ value of the following frame, is the value of $\theta_{1}+\Delta \theta_{1}$. If $\theta_{1}$ is equal to HLB $+\Delta \theta_{1}$, then the next frame is operated; if not, it is fed back to the part that computes $\rho_{1}$.

Fig 9 shows the position of the centerline in the $\mathrm{x}$ and $\mathrm{y}$ planes, and the forward image boundary of the left row. $\rho_{1}$ is the vertical distance between the origin and the centerline, and $\theta_{1}$ 
is the vertical angle between the y-axis and the centerline.

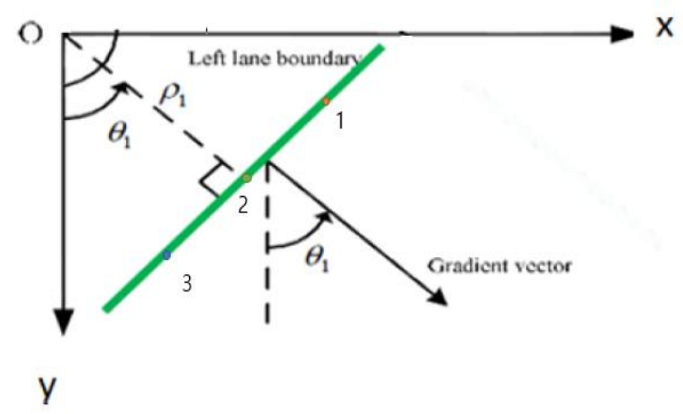

Fig.9 The lane distance in the $(\mathrm{x}, \mathrm{y})$ plane.

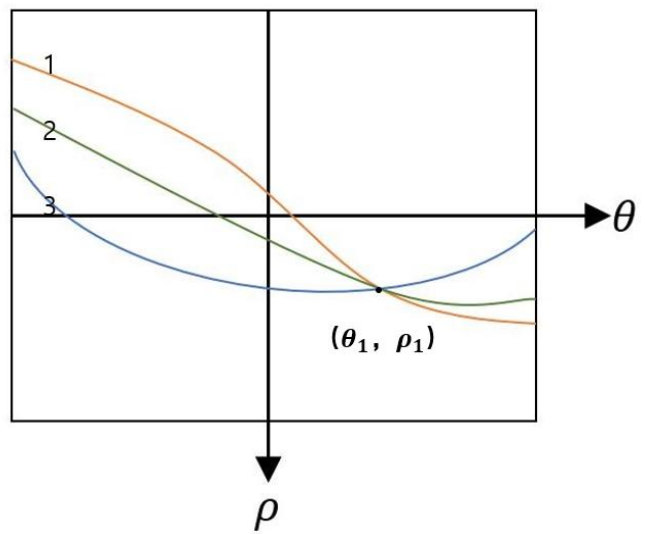

Fig.10 Hough space for lane detection with accumulator cells.

Fig 10 shows the hough area for lane detection with cumulative. The range of $\rho$ and $\theta$ values corresponding to the $\mathrm{x}$-axes and $\mathrm{y}$-axes of the hough area is $-90^{\circ} \leq \theta \leq$ $90^{\circ}$ and $-D \leq \rho \leq D$, and $D$ is the maximum diagonal length relative to the origin in the plane $x, y$.

The proposed algorithm only detects straight lines within the entire hough area, limiting only certain areas for centerline detection. In this process, the algorithm was implemented by setting only the angle value $\theta$. In the case of distance value $\rho$, it was not used as a variable in the implementation of the algorithm because in all frames within ROI, straight lines of different lengths detected within a certain angle can be determined as centerlines. When setting the distance value as a variable to reduce the range of cumulative cells, it may not be recognized as a centerline if only a certain portion of the line is spanned in a particular frame, or because of the computational speed decreases in the process of averaging multiple lines from one frame to one straight line behind, thus increasing algorithm efficiency. For HL-ROI and LL-ROI, only the left side of the lane is recognized and detected. Thus, to apply the hough conversion algorithm only two areas within the range where the left lane is expected to be located in the entire hough area, straight lines with $\theta$ values between LLB and HLB are extracted. In this algorithm, the LLB is set to $35^{\circ}$ and the HLB is set to $55^{\circ}$ to detect all the straight lines recognized within this range.

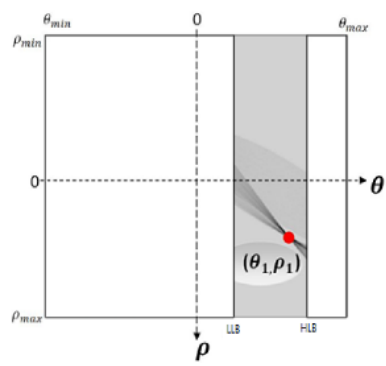

(a) hough space

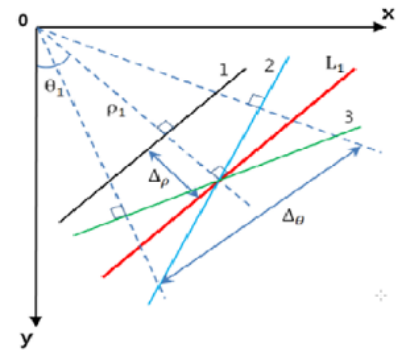

(b) $x-y$ space
Fig.11 The left lane optimized with accumulator cells.

Fig 11 (a) shows the optimization of cumulative cells for the left lane. Assuming $\Delta \theta_{1}$ between LLB and HLB based on $\theta_{1}$, many straight lines are detected within this range. Fig 11 (b) shows a diagram found in the left lane.

The conventional Hough transformation algorithm detected all the straight lines in the image, but it detected both lanes for the entire ROI through the cumulative cell. Also, in this paper, the ROI is divided into two units and HL-ROI and LL-ROI are detected and output in parallel. Fig 12 shows the result images after cumulative cells applied.
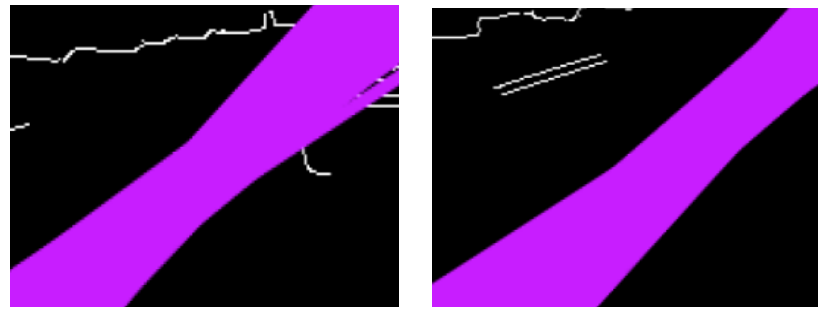

Fig.12 The result of after applied accumulator cells method.

In fig 12, the number of straight lines are output, and only one straight line can be determined by averaging output values. The mean values of $\Delta \theta_{1}$ is calculated using equation (3).

$$
\theta_{1}=\frac{1}{n} \sum \theta_{i 1}, \quad L L B \leq \Delta \theta_{1} \leq H L B
$$

Where $\theta_{i 1}$ is the $\theta$ of $n$ straight lines detected in the range of $\Delta \theta_{1}$. It is the $\theta$ value of straight lines. The result values of calculation are substituted for equation (2) to derive equation (4) as shown below.

$$
\rho_{1}=x \cos \left(\theta_{1}\right)+y \sin \left(\theta_{1}\right)
$$

Finally, the left lane is detected in HL-ROI and LL-ROI through equation (4).

\section{EXPERIMENTAL RESULT}

This article is the detection and recognition of the center line of the lane. For verify the effectiveness and robustness of the algorithm in this article, the driving vehicle collects road information. The actual shooting can ensure that the roads, vehicles, and trees in the video have rich colors Changes to 
ensure that the algorithm has stronger robustness.

This article configures Opencv3.4.1 on the Microsoft Visual Studio 2019 program platform for program simulation. With an image size of $640 * 480$ pixels, a real-time processing speed of 27 frames per second is achieved. After statistics of the results under various road conditions, the accuracy rate can reach $97.82 \%$. Through the detection results of various central lines, it is known that the method in this paper can stably recognize the central lines of various complex roads ahead. The detection results are shown in the figure.

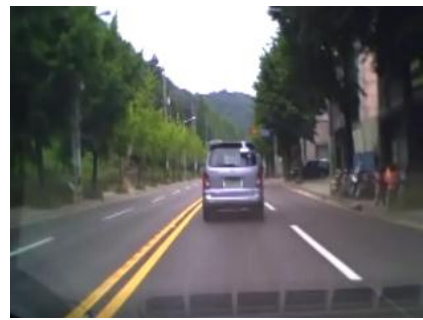

a. $R G B$ input image

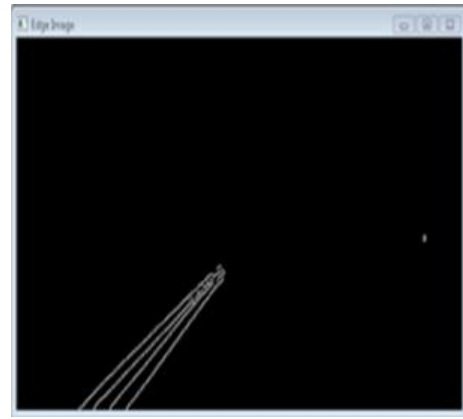

c. Edge detection

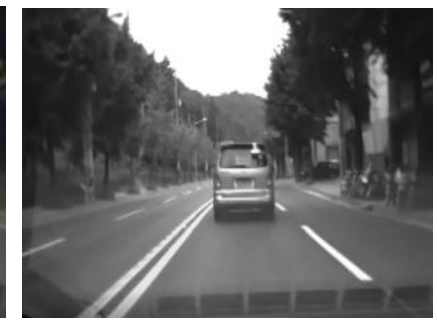

b. Grayscale and filter

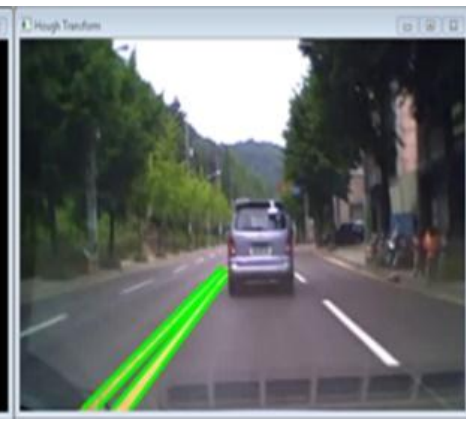

d. centerline recognition
Fig.13 The result of centerline recognition.

\section{CONCLUSION}

Aiming at the problem of the complex and changeable image acquisition environment, this paper uses the $45^{\circ}$ Sobel operator to extract the edge of the image. After acquiring the highquality image, the central line detection is performed through the improved Hough transform, which greatly improves the stability of the algorithm. Compared with traditional methods, this algorithm is more practical, improves the reliability and robustness of recognition, and has better application value in vehicle departure warning and other assisted driving research. But at present, simulation optimization is mainly carried out through simulation, and it needs to be transplanted to the hardware platform for practical application in daily life. The research will be further improved in the future.

\section{ACKNOWLEDGEMENTS}

This work was supported by Korea Hydro \& Nuclear Power company through the project "Nuclear Innovation Center for Haeoleum Alliance" and "Industrial technology infrastructure building innovation project " through the Korea Institute for Advancement of Technology (Grant Number: P0014572)

\section{REFERENCES}

[1] Cao Yuehua, Luo Wenguang, Lan Hongli, Zhao Xiaodong. Research on road lane line recognition algorithm in complex environment[J].Modern Electronic Technology, 2017, 40(14): 109-113.

[2] Li Yadi, Huang Haibo, Li Xiangpeng, Chen Liguo. Night lane detection based on Canny operator and Hough transform[J]. Science Technology and Engineering, 2016, 16(31): 234-237, 242.

[3] Zhang Kaixin, Xu Meihua.Target detection and recognition of lane departure real-time warning system[J].Journal of Xi'an Technological University, 2016, 36(7): 539-544, 572.

[4] V S BOTTAZZI, PVK BORGES, J JO. A vision-based lane detection system combining appearance segmentation and tracking of salient points[J]. $2013,36(1)$ : 443-448.

[5] Wang Xiaojin, Wang Zengcai, Zhao Lei. Lane line recognition based on vanishing point detection and segmented straight line model[J]. Mechatronics, 2017, 23(5): 41-46.

[6] Liu Yugang, Wang Zhuojun, Liu Yanfang, Zhang Zutao, Xu Hong. Reversing obstacle detection preprocessing method based on binocular vision image[J].Journal of Chongqing Jiaotong University (Natural Science Edition), 2018, 37(3): 92-98.

[7] Li Fei, Bin Yang, Luo Wenguang, Zhu Yingkai. Lane Line Detection Based on TMSDM6437[J]. Journal of Guangxi University of Science and Technology, 2018, 29(1): 43-48.

[8] Sui Liang,Dang Jianwu,Wang Yangping.Rapid lane line detection based on segment switching model[J]. Computer Applications and Software, 2017, 34(8): 201-205.

[9] D SEOK JO. Inverse perspective mapping based road curvature estimation[C]. International Symposium on System Integration, IEEE, 2014.

[10] X Du, K K Tan. Vision-based approach towards lane line detection and vehicle localization $[\mathrm{J}]$. Machine Vision and Applications, 2016, 27(2): 175-191.

[11] J.-H. Cho, Y.-M. Jang, and S.-B. Cho, "A High-performance Lane Recognition Algorithm Using Word Descriptors and A Selective Hough Transform Algorithm with Four-channel ROI," Journal of the Institute of Electronics and Information Engineers, vol. 52, no. 2, pp. 148-161, Feb. 2015.

[12] Ji-Hue Bae and Jae-Bok Song, "Monocular Vision Based Lane Detection Using Segmented Regions from Edge Information," 2011 IEEE, 8th International Conference on Ubiquitous Robots and Ambient Intelligence (URAI), pp. 499-502, Incheon, Korea, Nov. 2011.

[13] T. Wang, "Phd Forum: Real-Time Lane-Vehicle Detection for Advanced Driver Assistance on Mobile Devices," 2017 IEEE International Conference on Smart Computing (SMARTCOMP), Hong Kong, 2017, pp. 1-2, doi: 10.1109/SMARTCOMP.2017.7947034.

[14] J. Canny, "A computational approach to edge detection, "IEEE Trans. Pattern Anal. Mach. Intell” vol. PAMI-8, no. 6, pp. 679-698, Nov. 1986.

[15] W. Farag and Z. Saleh, "Road Lane-Lines Detection in RealTime for Advanced Driving Assistance Systems," 2018 International Conference on Innovation and Intelligence for Informatics, Computing, and Technologies (3ICT), Sakhier, Bahrain, 2018, pp. 1-8, doi: 10.1109/3ICT.2018.8855797.

[16] www.koroad.or.kr.

[17] www.opencv.org 